\title{
Kernos
}

Revue internationale et pluridisciplinaire de religion grecque antique

30 | 2017

Varia

\section{La déesse Korè-Perséphone : mythe, culte et magie en Attique}

Delphine Ackermann

\section{(2) OpenEdition \\ Journals}

Édition électronique

URL : http://journals.openedition.org/kernos/2508

DOI : 10.4000/kernos.2508

ISSN : 2034-7871

Éditeur

Centre international d'étude de la religion grecque antique

Édition imprimée

Date de publication : 1 octobre 2017

Pagination : 315-320

ISSN : 0776-3824

Référence électronique

Delphine Ackermann, «La déesse Korè-Perséphone : mythe, culte et magie en Attique », Kernos [En ligne], 30 | 2017, mis en ligne le 01 octobre 2017, consulté le 10 décembre 2020. URL : http:// journals.openedition.org/kernos/2508 ; DOI : https://doi.org/10.4000/kernos.2508

Ce document a été généré automatiquement le 10 décembre 2020.

Kernos 


\title{
La déesse Korè-Perséphone : mythe, culte et magie en Attique
}

\author{
Delphine Ackermann
}

\section{RÉFÉRENCE}

Alexandra DIMou, La déesse Korè-Perséphone: mythe, culte et magie en Attique, Turnhout, Brepols, 2016. 1 vol. 15,6 × 23,4 cm, 551 p. (Recherches sur les Rhétoriques Religieuses, 18). ISBN : 978-2-503-56508-8.

1 Fruit d'une thèse de doctorat, cet ouvrage s'inscrit dans la lignée des monographies consacrées à une divinité. Il vient combler une lacune de la recherche, puisque KoréPerséphone a été étudiée jusqu'à présent surtout en association avec Déméter, dans des travaux portant sur d'autres cités ou régions. L'A. a choisi de restreindre son enquête à l'Attique, en raison de l'abondance des sources, tout en adoptant la chronologie la plus vaste possible (toute l'Antiquité). Toutefois, elle n'hésite pas à sortir de cette zone géographique pour éclairer certains points de son sujet.

2 L'A. cherche à cerner la figure que revêtait la déesse en Attique et les fonctions qu'elle occupait tant au sein de la religion civique que des pratiques de dévotion et croyances privées. Elle s'appuie sur une abondante bibliographie et sur des sources antiques nombreuses et variées (littéraires, épigraphiques, papyrologiques), réunies pour la plupart dans un corpus comprenant le texte grec et la traduction. Elle tient compte également des dernières découvertes archéologiques, mais ne fait que peu de place à l'iconographie.

Dans une première partie, l'A. examine les versions du mythe de la déesse se référant à l'Attique, d'Homère aux néoplatoniciens, en passant par la tragédie et les textes orphiques. Elle étudie également les différentes images de la déesse dans les sources littéraires, ainsi son identification à la Lune, astre qui accueille les âmes des morts selon divers courants philosophiques, croyance que l'on trouve aussi dans des papyrus magiques et des épigrammes funéraires. 
4 Particulièrement attentive aux origines des mots, A.D. consacre deux chapitres aux différentes étymologies proposées par les Anciens pour les noms de Koré et de Perséphone. Quoique fantaisistes pour la plupart, elles permettent de mieux cerner les divers aspects de la déesse. Cette question est particulièrement difficile à traiter dans le cas de Perséphone, dont le nom a revêtu plusieurs formes, comme Perséphonéia, Pherséphonéia, ou encore la forme attique Pherrhéphatta. Ainsi, les étymologies la lient à la mort et au meurtre, au mythe de son rapt, à l'agriculture et à la végétation, à la lumière, à la sagesse. Pour Koré, l'exercice est plus simple, puisque ce nom la désigne comme la fille de Déméter. Frappée par la fréquence de l'association entre Perséphone et le meurtre (phonos), A.D. pose une question intéressante: Perséphone est-elle une tueuse ? Elle répond de manière contradictoire: dans un premier temps, elle estime que la vision d'une Perséphone terrifiante et meurtrière, et l'étymologie qui la rattache au meurtre, émanent des sources byzantines; si la déesse est une tueuse dans l'Antiquité, ce n'est qu'une tueuse de la végétation, vision métaphorique que l'on trouve notamment dans l'orphisme. Mais elle reconnaît ensuite que Perséphone est, comme les autres divinités souterraines, capable d'infliger le mal, pour venger une injustice ou tout simplement pour nuire à un ennemi: elle apparaît ainsi dans les tablettes de malédiction attiques, mais aussi dans les Choéphores d'Eschyle ou dans l' Électre de Sophocle. Il est étonnant que l'A. ne mette pas à profit ici l'étymologie proposée dans l'Hymne orphique à Perséphone, qui figure pourtant dans son corpus (T 210) : «Pherséphonè : car à jamais tu fais vivre (pherbeis) et mourir (phoneueis) toutes choses. » Toute erronée qu'elle soit, elle me semble illustrer au mieux l'ambivalence de la déesse, à la fois pourvoyeuse de vie et de mort, pour la végétation comme pour les hommes.

Dans un dernier chapitre, les noms de la déesse utilisés dans le culte en Attique sont passés en revue. A.D. remarque que Koré-Perséphone est appelée très rarement Pherrhéphatta ou, bien plus souvent, Koré, et qu'elle est la plupart du temps couplée avec Déméter. Elle souligne l'usage fréquent du duel pour la mère et la fille, par exemple dans les serments. Le lecteur reste sur sa faim : on aurait aimé une tentative d'explication de l'appellation Pherrhéphatta dans un contexte cultuel, remarquable puisqu'elle est la forme attique de Perséphone, or en principe, dans la religion grecque, les dieux infernaux ne sont pas vénérés sous leur vrai nom mais sous une forme anonyme, euphémistique ou neutre.

6 Dans une deuxième partie, la plus substantielle, l'A. analyse les fêtes et cultes où Koré apparaît, seule ou avec Déméter, dans la cité et les dèmes, à savoir les Thesmophories, les Halôa, les Petits et Grands Mystères, les Skira, les autres cultes locaux (où le culte éleusinien est parfois venu se greffer sur des rites plus anciens) et les cultes sur l'Agora d'Athènes. À chaque fois, elle propose une étude étymologique du nom de la fête, expose le déroulement de la cérémonie, suit l'évolution de la fête à travers le temps et compare avec les mêmes fêtes hors de l'Attique. Cela lui permet d'établir une géographie du culte de Koré-Perséphone, d'en faire ressortir les particularités selon les lieux où il est pratiqué et de mettre en lumière le rôle de la déesse pour la cité d'Athènes. A.D. consacre en outre un intéressant chapitre au rapport de Déméter et Koré avec la vie politique, où on les voit agir comme alliées des Athéniens dans les batailles ou permettre que leurs fêtes soient perturbées à des fins de stratégie militaire.

7 À part quelques exceptions (ainsi la vénération de Déméter et Koré dans les ateliers de traitement du métal de la région du Laurion), les conclusions de cette longue partie 
sont sans surprise : le culte éleusinien et le culte thesmophorique sont les «deux axes principaux " du culte de Koré-Perséphone en Attique ; associée à sa mère, Koré est vénérée surtout dans le cadre de fêtes féminines, qui promeuvent la fertilité de la terre et la fécondité des femmes; dans les Mystères, elle offre aux mystes la promesse d'un meilleur au-delà à travers l'initiation. L'image de la déesse dans le culte n'est donc pas effrayante, car c'est sous son aspect de Koré qu'elle est adorée et non en tant que souveraine des morts ; là encore, l'A. élude le cas de Pherrhéphatta, qui disposait d'un sanctuaire sur l'Agora. Les différents chapitres sur ces fêtes bien connues auraient pu être beaucoup plus courts; sous cette forme, on se demande s'ils sont vraiment nécessaires, d'autant plus que la bibliographie n'est parfois pas à jour (c'est le cas entre autres pour les Mystères d'Éleusis). L'enquête aurait peut-être gagné en originalité en distinguant davantage les différents niveaux de la pratique cultuelle athénienne (à cet égard, il est discutable de placer les cultes des dèmes sous le chapeau de "culte civique ») : cela permettrait de mieux mettre en évidence l'articulation entre le culte civique des deux déesses à Éleusis et à Athènes, et leur culte dans les dèmes, une question particulièrement intéressante qui n'est abordée que superficiellement. Par ailleurs, l'A. aurait pu s'attacher davantage à l'étude du personnel cultuel desservant Koré (et bien souvent Déméter) en Attique, en mettant en avant le rôle des génè.

Quelques remarques sur les cultes locaux : il faut enlever le dème de Képhalè de la liste, puisque la borne de sanctuaire invoquée vient en réalité de Thorikos ; A.D. laisse planer le doute inutilement. Par ailleurs, elle a tendance à voir des cultes à mystères là où ils ne sont pas attestés, par ex. à Thorikos et à Phrearrhioi : la présence d'un culte éleusinien ne signifie pas forcément que l'on y pratiquait des mystères.

9 Dans la troisième et dernière partie, l'A. étudie la figure de Koré-Perséphone dans la sphère privée, à travers trois thèmes: les pratiques magiques (dont témoignent les tablettes d'envoûtement, particulièrement nombreuses à Athènes, et les papyrus magiques), les associations religieuses (il est surtout question dans ce très court chapitre de celle des Iobacchoi) et l'interprétation des rêves (d'après la Clef des songes d'Artémidore). C'est sans aucun doute la partie la plus originale de l'ouvrage, la figure de Koré-Perséphone n'ayant été que peu étudiée dans les pratiques magiques et dans l'interprétation des rêves.

Dans les pratiques magiques, Perséphone figure parmi les divinités souterraines les plus fréquemment invoquées. Elle apparaît comme une divinité vengeresse et redoutable, capable de nuire à autrui. Koré est attestée elle aussi à quelques reprises dans un contexte magique, phénomène surprenant, que l'A. ne tente pas d'expliquer. A.D. se lance ensuite dans une démonstration de ce qui oppose les pratiques magiques aux pratiques religieuses civiques, en s'appuyant pour ces dernières sur les dédicaces de femmes et les décrets en l'honneur de prêtresses exposés dans les sanctuaires, notamment dans l'Éleusinion de l'asty. L'analyse ne tient pas assez compte des particularités propres à chaque catégorie de documents, ce qui fausse une partie des observations; et pourquoi ne retenir que les inscriptions de l'Éleusinion mentionnant des femmes? La grille de lecture genrée n'apporte rien ici.

11 Le chapitre sur la figure de Koré-Perséphone dans l'onirocritique amène l'A. à sortir des sources athéniennes. L'érudition d'Artémidore offre un mélange de concepts philosophiques, de croyances et de pratiques religieuses qui permet de compléter l'image de la déesse en Grèce. Par ex., Artémidore se réfère à l'aspect guérisseur de la déesse (avec Déméter), particulièrement pour les yeux; cet aspect se retrouve dans le 
culte éleusinien, dans les textes orphiques et dans l'association des deux déesses à Asklépios dans divers sanctuaires en Attique et ailleurs. Le nom même de Koré la rattache à la vue par une fausse étymologie (korè désigne aussi la pupille de l'œil). A.D. pose l'hypothèse convaincante d'un lien avec l'importance de la vue et de la lumière dans les Mystères.

12 A.D. traite dans un appendice la figure de Koré-Perséphone chez le néoplatonicien Porphyre. Des aspects moins connus de la déesse apparaissent, comme l'association de Perséphone à la colombe sauvage, l'image de Koré comme tisserande ou encore sa qualification de « douce comme le miel » (Melitôdès).

13 S'ensuit le corpus, riche de 394 textes, et une section «Cartes et plans » comprenant 4 illustrations. Sur la carte de l'Attique (pl. I) où sont situés les lieux dans lesquels un culte de la déesse est attesté, la position des dèmes est obsolète, car elle est reprise de la vieille étude d'A.Milchhöfer de 1892 (Untersuchungen über die Demenordnung des Kleisthenes), or la topographie des dèmes a été passablement modifiée depuis, grâce aux travaux de J.S. Traill entre autres. Par ailleurs, il aurait fallu mettre une marque de doute à côté des dèmes dans lesquels un culte n'est pas certain (comme Korydallos) et enlever les fausses occurrences (comme Képhalè).

Plusieurs index viennent compléter le livre. Hélas, l'index des sources est rendu en grande partie inutilisable par de nombreuses erreurs de montage : on trouve ainsi des auteurs antiques dans les rubriques "inscriptions", "papyri » et "scholies ", et des inscriptions dans la rubrique " papyri ».

De cette longue enquête, ressortent deux aspects de la déesse qui me paraissent particulièrement intéressants, à savoir sa dualité et sa faible individualité. KoréPerséphone est en effet presque toujours associée à Déméter, que ce soit dans la mythologie et dans les rites, et son nom même de Koré la définit comme fille de Déméter. Elle ne prend son indépendance que dans quelques endroits de l'Attique: dans le dème de Teithras où se trouvait un Koreion, peut-être dans le dème de Korydallos, s'il existait bien un culte de Koré Sôteira, et dans le Pherrhéphattion de l'Agora. La déesse apparaît aussi sans Déméter dans le domaine de la magie, où l'appellation Perséphone domine largement. Quant à la dualité de Koré-Perséphone, elle est déjà bien visible dans les deux noms qu'on lui prête: Perséphone, reine des Enfers et épouse d'Hadès, pourvoyeuse de mort; Koré, fille de Déméter, pourvoyeuse de vie et de fertilité, et maîtresse avec sa mère des fameux Mystères d'Éleusis. Il manque dans cette étude un chapitre sur l'usage différencié de ces deux dénominations dans les sources antiques : au-delà des exigences de la métrique et de la variatio, la tendance générale est de nommer la reine des Enfers Perséphone et la fille de Déméter Koré, mais il existe des exceptions troublantes, comme l'apparition de Koré dans les pratiques magiques, ou l'usage de l'appellation Pherrhéphatta dans le culte. Il aurait été utile de clarifier cette question dès le début, car A.D. parle souvent de Perséphone ou de Koré indifféremment, et parfois de Koré-Perséphone, évidemment pour varier le vocabulaire, mais cela peut donner au lecteur l'impression que les deux dénominations sont interchangeables.

On regrette également l'absence d'une réflexion sur les épithètes de la déesse, où seraient soigneusement distinguées les épithètes cultuelles et les épithètes poétiques. Certes, quelques épithètes poétiques sont relevées çà et là, comme epainè, arrhètos, kallipais ou hagnè, ainsi que quelques épiclèses (Thesmophoros, partagée avec Déméter ; Prôtogonè pour Koré seule dans le dème de Phlya), mais sans développement 
approfondi. Par ailleurs, il faut rejeter Koré Kourotrophos que l'A. croit lire dans la dédicace $I G \mathrm{II}^{2}$, 4778. Je signale une défixion qui semble avoir échappé à l'A., où Perséphone est qualifiée de kalôpis (E. ZIEBARTH, Neue Verfluchungstafeln $25 \mathrm{~B}, 1.11$ ); ce texte aurait pu alimenter la réflexion sur les liens entre la déesse et l'œil.

Des problèmes importants se posent dans le traitement des sources. Les inscriptions en particulier sont malmenées :

18 - l'A. utilise souvent des éditions obsolètes, par ex. pour les inscriptions d'Éleusis, où l'on trouve avec surprise des références au vieux corpus IG $\mathrm{I}^{2}$, au lieu de celui de K. Clinton qui fait aujourd'hui autorité ;

19 - l'A. se méprend parfois sur la nature du texte: des dédicaces sont prises pour des décrets; IG II ${ }^{2}, 1437$ n'est pas une "inscription sur une hydrie " (p. 97) mais un inventaire des trésoriers d'Athéna dont une section concerne des hydries;

20 - des textes sont pris pour des inscriptions différentes alors qu'il s'agit de la même mais dans diverses éditions : ainsi pour le fameux calendrier sacré d'Athènes des alentours de 400, pour lequel l'édition de référence de S.D. LAMBERT, ABSA 97 (2002), p. 353-399 (SEG 52, 48) n'est pas mentionnée ;

21 - dans le corpus, certaines inscriptions ne sont reproduites que très partiellement (jusqu'à trois mots seulement !), alors qu'elles sont commentées dans leur intégralité, le lecteur ne pouvant vérifier l'interprétation de l'A. (par ex. la défixion T 370; par ailleurs, le même support comportait une autre défixion, non mentionnée par l'A., pourtant Perséphone est mentionnée à la 1. 14).

22 Ces négligences sont particulièrement graves lorsque les sources épigraphiques sont les seuls témoignages, ainsi pour les attestations du nom de Pherrhéphatta, dont le nombre est à réduire si l'on enlève les restitutions anciennes désormais rejetées et les occurrences issues en réalité d'une même inscription.

23 Bon nombre des traductions que propose l'A. sont fautives, que ce soit pour les sources épigraphiques (ex. T 342 : c'est la "prêtresse d'Athéna », et non la « déesse d'Athéna »; T 345 : c'est «à la prêtresse de Pluton », et non « de la prêtresse de Pluton »; T 373 : ce n'est pas «ériger la stèle à Koreion» mais «dans le Koreion »; T 374: "ayant été gymnasiarque » et non " étant gymnasiarque ») ou littéraires (ex. T 162 : Daeira n'est pas "gardienne de Perséphone et de Pluton», mais "gardienne de Perséphone sous l'autorité de Pluton»; p. 180 : le dadouque ne portait pas "une barbe " d'après Plutarque mais un bandeau [strophion], la bonne traduction, tirée de la CUF, figurant pourtant dans le corpus sous le $\mathrm{T}$ 105).

24 Une mauvaise interprétation des sources conduit inexorablement l'A. à échafauder des raisonnements qui ne tiennent pas. Je ne donnerai que quelques exemples :

25 - p. 124 n. 80, A.D. dit que «le culte de Kalligeneia a été confirmé dans le cadre éleusinien ", citant cinq inscriptions, or il s'agit de dédicaces à Kourotrophos, et dans un cas d'une borne d'un sanctuaire de Zeus Kataibatès ;

26 - p. 174-175, sur la base de l'inscription I.Eleusis 196, 1. 10-11, l'A. développe l'hypothèse d'une ouverture des Halôa aux deux sexes sous Démétrios Poliorcète, ajoutant que " sous le règne de Démétrios (...), les changements dans les rituels des cultes n'étaient pas rares»(p.175), exemples à l'appui; or il s'agit de Démétrios II dans cette inscription ; 

sacrifiée à "Koré fille de Déméter». A.D. en déduit que dans cette pièce, Koré « est investie du rôle de Perséphone en tant que déesse du monde d'en bas, rôle qui peut être en relation avec l'aspect chtonien de Koré dans le culte de Marathon » (p.52). Elle pense en effet que dans le calendrier sacré du dème de Marathon, il y a " cohabitation " de Daeira (identifiée avec Perséphone ou du moins étroitement associée à la déesse), Déméter Achaia et Koré. Or on ne peut parler de cohabitation que si les trois déesses sont vénérées au même endroit à la même occasion, ce qui n'est pas le cas : seules Déméter Éleusinia et Koré sont associées dans un sacrifice fait par les démotes de Marathon, sacrifice dont A.D. ne discute étrangement pas, alors qu'elle analyse les sacrifices pour Daeira et pour Déméter Achaia. Par ailleurs, A.D. ne tient pas compte du fait que si Euripide parle de Koré et non de Perséphone à cet endroit, c'est surtout pour répondre aux exigences du genre poétique ;

- p. 134-135, à propos des Thesmophories du dème d'Halimonte, vues parfois comme un prélude aux Thesmophories de l'asty car elles ont lieu la veille : selon A.D., la veille des Thesmophories de l'asty, les Halimousiennes feraient le déplacement de la ville vers le dème pour remplir leurs obligations religieuses, puis reviendraient dans l'asty pour participer aux Thesmophories de la ville "puisque c'était là que leur habitation principale était située » (p.135), citant à l'appui de cette hypothèse le C. Euboulidès de Démosthène. Or ce discours montre exactement le contraire, à savoir que les démotes d'Halimonte habitaient encore dans leur dème pour la plupart. Relevons encore une erreur à ce sujet : l'A. pense que les Thesmophories d'Halimonte se déroulaient dans le sanctuaire de Déméter au cap Côlias, mentionné par Plutarque et Hésychius notamment ; or ce cap fait partie du dème de Phalère.

À ces problèmes de fond s'ajoutent des problèmes de forme : l'édition de l'ouvrage est bâclée, ainsi qu'en témoignent les nombreuses coquilles, les erreurs dans les renvois aux notes ou aux textes du corpus, le manque d'uniformisation entre les traductions de l'A. données dans le texte et celles d'un autre traducteur reproduites dans le corpus (ex. p. 35 et $\mathrm{T} 209 ;$ p. 84 et $\mathrm{T} 44$ ), les parties du texte grec retranscrit dans le corpus non traduites (ex. T 88, T 161, T 250, T 362 B), les traductions prises chez d'autres auteurs ne correspondant pas au texte grec reproduit dans le corpus (ex. : T 352).

En définitive, cet ouvrage relève plutôt de la synthèse que de la thèse : A.D. rassemble scrupuleusement les sources antiques et modernes, mais elle n'en tire pas vraiment de nouveautés. Elle s'arrête la plupart du temps au stade du constat, sans tentative d'explication, ou se contente d'exposer les différentes théories existantes, sans les confronter ni prendre position. Ses rares hypothèses personnelles sont souvent fondées sur une mauvaise compréhension des sources. Enfin, une question primordiale n'est pas abordée frontalement, à savoir si la figure de Koré-Perséphone en Attique est un vrai sujet : est-il pertinent d'individualiser la figure de la déesse dans cette région, ou n'est-elle finalement pas différente de celle que l'on trouve ailleurs dans le monde grec? 


\section{AUTEURS}

DELPHINE ACKERMANN

Université de Poitiers 